\title{
Diffusion and superdiffusion of a quantum particle in time-dependent random potentials
}

\author{
J. Heinrichs \\ Institut de Physique B5, Université de Liège Sart Tilman, B-4000 Liège, Belgium
}

Z. Phys. B 89, 115 (1992)

We first wish to correct a mistake in our analysis of the evolution of a quantum particle in a random potential which slightly modifies our earlier conclusions concerning the effect of a finite correlation time $\tau$ in the asymptotic domain $t \gg \tau$. In Eqs. (16) and (16.a), which follow from (15), the integrand of the K-integral in the terms proportional to $\tau$ should be $K g(K)\left\langle\tilde{\rho}\left(k^{\prime}+K, k+K, s\right)\right\rangle$ instead of $g(K)\left\langle\tilde{\rho}\left(k^{\prime}+K, k+K, s\right)\right\rangle$. No misprint has occured in (19), but in (21) the term proportional to $\tau$ should be replaced by

$$
\begin{aligned}
& -\frac{i \tau V_{0}^{2}}{2 \hbar m} \int_{-\infty}^{\infty} \frac{\mathrm{d} K}{2 \pi} \int_{-\infty}^{\infty} \frac{\mathrm{d} k^{\prime \prime}}{2 \pi} k^{\prime \prime}\left(K+k^{\prime \prime}\right) g\left(K+k^{\prime \prime}\right) \\
& \quad \times\langle\tilde{\rho}(-K,-K, s)\rangle .
\end{aligned}
$$

Also, in using (16.a) we have employed $-K-k^{\prime \prime}$ (and not $K-k^{\prime \prime}$, as was stated) as new variable in the $\mathrm{K}$-integrals.

For a spatially correlated random potential, the expression (A.21) leads to an additional term, $-\tau V_{0}^{2} G^{\prime \prime}(0) /$ $m^{2} s^{3}$, on the r.h.s. of (23) and to a correction (for the domain $t \gg \tau),-\tau V_{0}^{2}\left(2 m^{2}\right)^{-1} G^{\prime \prime}(0) t^{2}$ in the mean square displacement (29).

We note that our first principles results for the mean squared displacement and for the averaged kinetic energy given in (30) differ qualitatively from the recent analysis of Lebedev et al. [1] which reaffirms the earlier results of Golubovic et al. for the effect of a finite correlation time of the random potential.

For a spatially uncorrelated random potential, the effect of the correction (A.21) is to replace the factor $\left\langle\widetilde{E}_{\text {kin }}(s)\right\rangle$ in $(24)$ by $\left\langle\widetilde{E}_{\text {kin }}(s)\right\rangle-\tau V_{0}^{2} \delta^{\prime \prime}(0)(4 m s)^{-1}$, where the second term yields a further correction term, $-\tau V_{0}^{2}\left(m^{2} w_{0}^{2}\right)^{-1} \delta^{\prime \prime}(0)\left[1-2^{-1} \hbar^{-2} V_{0}^{2} \delta(0) \tau\right]\left(e^{-w_{0} t}-1\right.$ $\left.+w_{0} t\right)$ in (31). The asymptotic diffusion form of $\left\langle x^{2}(t)\right\rangle$ is then

$\left\langle x^{2}(t)\right\rangle=-\frac{2 \hbar^{4}}{m^{2} V_{0}^{2}} \frac{\delta^{\prime \prime}(0)}{(\delta(0))^{2}}\left[1-\left(\frac{V_{0}^{2} \delta(0) \tau}{2 \hbar^{2}}\right)^{2}\right] t$, which coincides with the result found earlier for $\tau=0$ $[2,3]$ and also, after performing a lattice-regularization of non-finite quantities $[2,4]$, with the well-known result for a tight-binding band with uncorrelated random site energies. The boundedness of the mean kinetic energy in the latter case is reflected in the saturating form of (32).

We take this opportunity to add a further comment. We have noticed that our lattice-regularization procedure for the case of a spatially uncorrelated potential (i.e. when $g(K)$ which acts as a convergence factor for various integrals, is replaced by unity) is not unique as far as the reduction of $\tau$-independent terms is concerned. We now discuss an alternative more straightforward regularization procedure. It consists in using systematically the symmetry property (see Sect. III)

$$
\begin{aligned}
\tilde{\Lambda}\left(k^{\prime}, k, s\right) & =\int_{-\infty}^{\infty} \frac{\mathrm{d} k^{\prime \prime}}{2 \pi}\left\langle\tilde{\rho}\left(k^{\prime}-k^{\prime \prime}, k+k^{\prime \prime}, s\right)\right\rangle \\
& =\tilde{\Lambda}\left(k^{\prime}+K, k+K, s\right)
\end{aligned}
$$

(together with the finite lattice versions of integrals $\left.\delta^{(n)}(0)=(2 \pi)^{-1} \int_{-\infty}^{\infty} k^{n} \mathrm{~d} k, n=0,2\right)$ at every step of the analysis of the mean squared displacement, $\left\langle x^{2}(t)\right\rangle$, for a $\delta$-correlated random potential. This generally assumes a reinterpretation of $\tilde{\Lambda}\left(k^{\prime}, k, s\right)$ on a discrete lattice since (A.2) is not necessarily obeyed for a continuum (as the integral defining $\tilde{\Lambda}$ may not converge), while being automatically verified on a finite lattice with periodic boundary conditions. The Eq. (A.2) is implied in our previous treatment for $\tau=0$ [2] where it plays, in fact, an important role since the final result for $\left\langle x^{2}(t)\right\rangle$ was found to coincide with an earlier expression for a finite lattice [5]. Its systematic use for studying corrections in $\left\langle x^{2}(t)\right\rangle$ at finite $\tau$ appears then most natural. In particular, we do not introduce the change of integration variable which was used in obtaining (19), (21) and (25) from (16.a) since this assumes regularization of the different integral which enters in the term proportional to $\tau$ in (16.a) when $g(K)=1$. In the present discussion, we propose, on the contrary, to 
regularize the effects of the $\tau$-term of (16.a) in Eqs. (19), (21) and (25) using only the lattice regularization (A.2), in addition to the redefinition of $\delta^{(n)}(0)$. Thus by using (A.2), the $\tau$-dependent term in the analog of (19) for $g(K)=1$ vanishes [instead of reducing to the value $-i \tau(\hbar m s)^{-1} V_{0}^{2}\langle\tilde{A}(s)\rangle$ obtained from (19)]. Next, the evaluation of the $\tau$-dependent term in the analog of (A.21) reduces to the same result as found above. Similarly, the use of (A.2) in (25) leads to (27). Finally, the present regularization scheme, based entirely on (A.2), yields an expression for $\left\langle x^{2}(t)\right\rangle$ which differs from (31) by the replacement of $m^{*}$ by $m$ and the addition of the term $-2 \hbar^{2} \tau \delta^{\prime \prime}(0)\left[2 m^{2} \delta(0)\right]^{-1}\left[w_{0}^{-1}\left(e^{-w_{0} t}-1\right)+t\right]$ on the r.h.s. For $w_{0} t \gg 1$ this leads to

$\left\langle x^{2}(t)\right\rangle=-\frac{2 \hbar^{4}}{m^{2} V_{0}^{2}} \frac{\delta^{\prime \prime}(0)}{(\delta(0))^{2}}\left[1+\frac{V_{0}^{2} \delta(0) \tau}{2 \hbar^{2}}\right] t$, i.e. a correction linear in $\tau$ in the diffusion constant instead of the quadratic term of (A.1). The mean kinetic energy is given by (32), which is thus independent of $\tau$ in the framework of our general study for small $\tau$ and for $t \gg \tau$.

\section{References}

1. Lebedev, N., Maass, P., Feng, S.: Phys. Rev. Lett. 74, 1895 (1995)

2. Heinrichs J.: Z. Phys. B 57, 157 (1984)

3 . In the course of preparing this erratum we detected an error in the earlier calculation [2] where the final result (18) must be divided by 2

4. Fischer, W., Leschke, H., Müller, P.: Phys. Rev. Lett. 73, 1578 (1994)

5. Madhukar, A., Post, W.: Phys. Rev. Lett. 39, 1424 (1977) 